\title{
High-Speed Visualization of Electrode Erosion in Multi-Phase Alternating Current Arc*
}

\author{
Manabu TANAKA**, Tomoki IKEBA**, Yaping LIU**, Sooseok CHOI** \\ and Takayuki WATANABE** \\ **Tokyo Institute of Technology, \\ 4259 Nagatsuta-cho, Midori-ku, Yokohama, Kanagawa 226-8502, Japan \\ E-mail:mtanaka@chemenv.titech.ac.jp
}

\begin{abstract}
A multi-phase AC arc plasma has been applied in the glass melting technology as a promising heat source. The electrode erosion of the multi-phase arc is one of the most important issues to be solved. In the present work, the high-speed camera system with appropriate band-pass filters was applied to visually observe the dynamic behavior of the electrode metal vapor in the arc on millisecond time scale. By using this system, the evaporation of $2 \%$-thoriated tungsten electrode was visually observed at the anodic period in the case of the 12-phase arc, while electrode evaporation is negligible in the 2-phase arc. High-speed observation of the oxygen entrainment in the arc was also carried out. Results indicated the ambient oxygen is entrained into the arc more easily with increasing the number of the phases. As a result, the electrode evaporation becomes more severe with larger number of the phases.
\end{abstract}

Key words: Thermal Plasmas, Multi Phase AC Arc, High Speed Camera, Electrode Phenomena, Electrode Erosion, Tungsten Electrode

\section{Introduction}

The glass industry is a large global industry that annually produces more than 100 million tons of glass products such as sheet glass, container glass, fiber glass, optical glass, and so on. Most glass has been produced by typical Siemens-type melter fired in air with heavy oil or natural gas as the fuel. This type of melter has been used for more than 140 years because of its good large-scale performance and continuous melting system. In the air-fuel fired furnace, the heat transfer from above burner flame to glass melt is so low that the conventional melting technology is energy intensive and time consuming, especially in the melting and the refining processes. With the rapid growth of glass usage and the increased energy and environment issues, it is crucial to develop a new glass melting technology to solve these problems.

An innovative in-flight glass melting technology with thermal plasmas was developed from the above point of view ${ }^{(1)-(4)}$. The granulated raw material with small diameter is dispersed in thermal plasma and the powders contact fully with the high temperature plasma. The high heat-transfer and temperatures of the plasma will melt the raw material quickly. At the same time, the decomposed gas of carbonates is removed during the in-flight treatment to reduce the refining time considerably. Compared with the traditional glass production which costs several days, the total vitrification time is evaluated as only $2-3 \mathrm{~h}$ at the same productivity as the fuel-fired melter.

The multi-phase AC arc is one of the most suitable heat sources for the in-flight glass melting because it possesses many advantages such as high energy efficiency, large plasma

${ }^{*}$ Received 1 Feb., 2013 (No. 13-0051) [DOI: 10.1299/jst.8.160]

Copyright $@ 2013$ by JSME 
volume (about $100 \mathrm{~mm}$ in diameter), low velocity $(5-20 \mathrm{~m} / \mathrm{s}){ }^{(5),(6)}$. Recent experimental works revealed the importance of the number of the phases on the discharge characteristics and the arc stability ${ }^{(7),(8)}$. Furthermore, the high-speed camera system was applied to investigate the electrode phenomena of the multi-phase AC arc ${ }^{(9)}$. However, the understandings of the multi-phase AC arc are still not enough for the practical use of the multi-phase arc system in glass melting process. Especially, electrode erosion is one of the considerable issues need to be solved.

Electrode erosion phenomena in the DC arc torches for the arc welding and arc cutting processes were found as a significant problem because it determined the lifetime of the electrode during the processes. In the case of the plasma cutting torch, hafnium is used as the cathode material because the oxygen gas is generally used in the plasma cutting processes. The effects of the arc current, pressure, shielding gas flow, and the cathode geometry on the hafnium erosion were reported ${ }^{(10)-(13)}$. Hafnium cathode erosion is dominated by the ejection of small liquid metal droplets ${ }^{(13)}$. The optimization of the initial cathode shape was tried to reduce the cathode erosion ${ }^{(14),(15)}$. In the case of the arc welding processes, the tungsten with metal oxide, such as thoria, is generally used as cathode because of its high melting point and low work function. The effects of the arc current, the plasma gas composition, the pressure, and the cathode's geometry on the cathode erosion were systematically studied ${ }^{(16)-(19)}$. In addition, the evaporation of the anode, which is the substrate of the welding process, has been studied. Theoretical and experimental efforts revealed the influence of the anode material addition into the arc ${ }^{(20),(21)}$. However, the electrode erosion phenomena in the AC arc torches have rarely been reported ${ }^{(22)}$.

In the previous work, the electrode temperature measurements of the multi-phase AC arc was conducted by using the high-speed camera system with the appropriate band-pass filters to understand the electrode phenomena in the multi-phase AC arc ${ }^{(9)}$. In the present work, the same system was applied to observe the dynamic behavior of the vapors in the arc on the millisecond time scale. The purpose of the present study is to visually observe the electrode erosion and to investigate the effect of the number of the phases on the electrode erosion in the multi-phase AC arc.

\section{Experimental}

\subsection{Experimental setup}

A schematic diagram of the experimental setup is shown in Fig. 1. It consisted of 12 electrodes, arc chamber, and AC power supply. The electrodes were made of tungsten $(98 \mathrm{wt} \%)$ and thoria $(2 \mathrm{wt} \%)$ with diameter of $6 \mathrm{~mm}$. City water was used to cool the electrodes at $3 \mathrm{slpm}$ of water flow rate for each electrode. The electrodes were divided into two layers, upper six electrodes and lower six electrodes. The upper electrodes were positioned at an angle of 30 degree with regard to the horizontal plane, while the lower electrodes were at an angle of 5 degree to control the plasma volume. The electrodes were symmetrically arranged by the angle of 30 degree as shown in Fig. 2. Because the melting points of tungsten oxides are lower than that of metal tungsten, 99.99\% argon was injected around the electrode at $5 \mathrm{slpm}$ of gas flow rate to prevent them from the oxidation. As the multi-phase arc discharge was generated under the atmospheric air except of the above mentioned argon, the plasma source gas was mainly air. 24 Sets of arc welding transformers with single-phase AC (DAIHEN B-300, Japan) were applied to generate the multi-phase AC arc discharge. More details about the 12-phase AC power supply were explained in the previous report ${ }^{(4)}$. In the present work, arc current was adjusted at AC $100 \mathrm{~A}$ for each electrode.

The applied voltage between each electrode and the neutral point of the coil of the transformer can be calculated by the following equation: 


$$
V_{i}^{N}=V_{m}^{N} \sin [\omega t-2 \pi(i-1) / 12], \quad(i=1,2 \ldots 12)
$$

Where $V_{i}^{N}$ indicates the applied non-load voltage for each electrode number $i$ and $V_{m}{ }^{N}$ indicates the amplitude of the non-load voltage (about $220 \mathrm{~V}$, AC50Hz). The voltage was applied to only electrode No. 1 and No. 7 for the 2-phase arc discharge. In the case of the 12-phase arc discharge, the voltage was applied to electrode No. 1, 2..12.

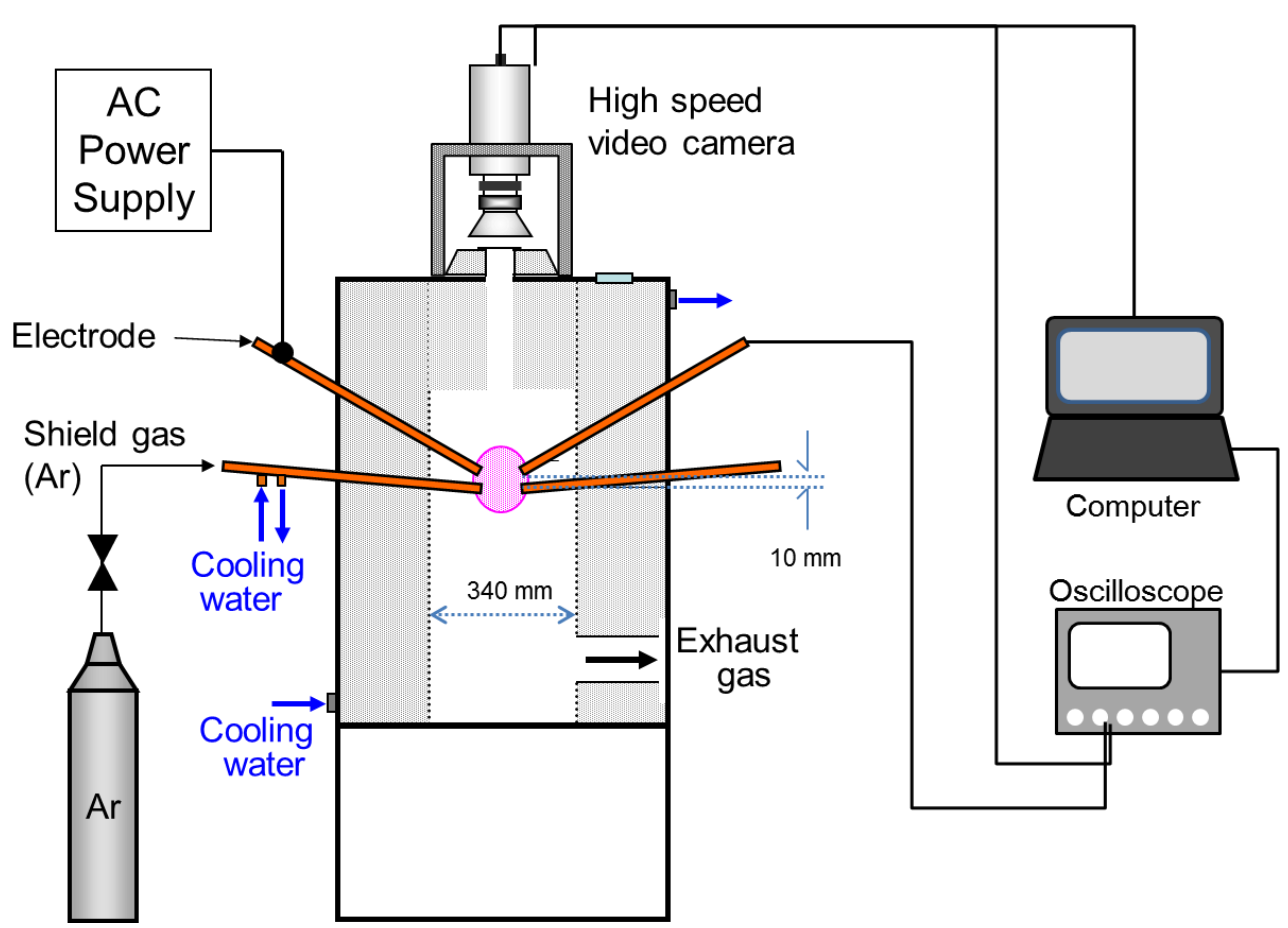

Fig. 1 Schematic illustration of the multi-phase AC arc.

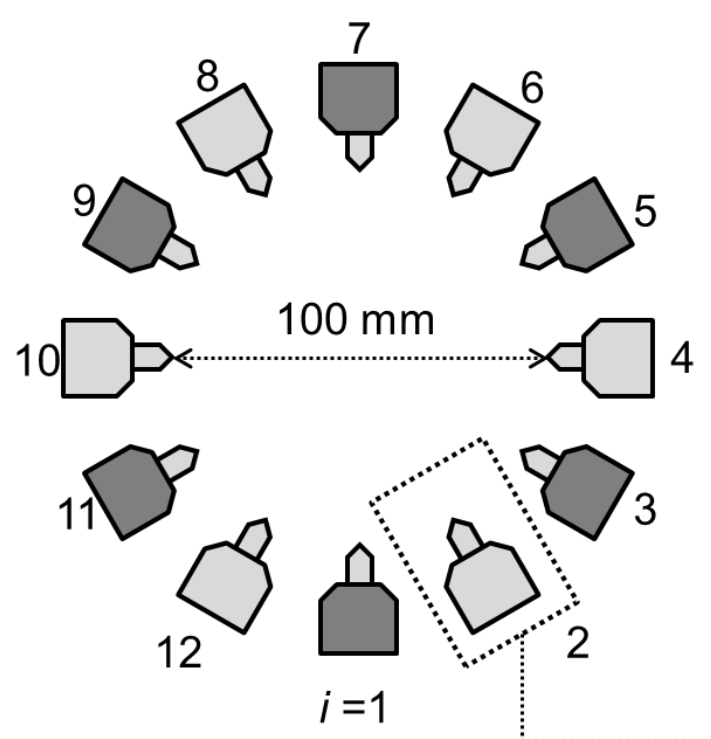

SUS jacket $\quad 2 \%-\mathrm{ThO}_{2} \mathrm{~W}$ electrode

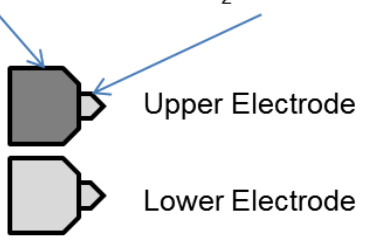

High-Speed Observation Region

Fig. 2 Cross section of the electrode region and the observation area by the high-speed camera system. 


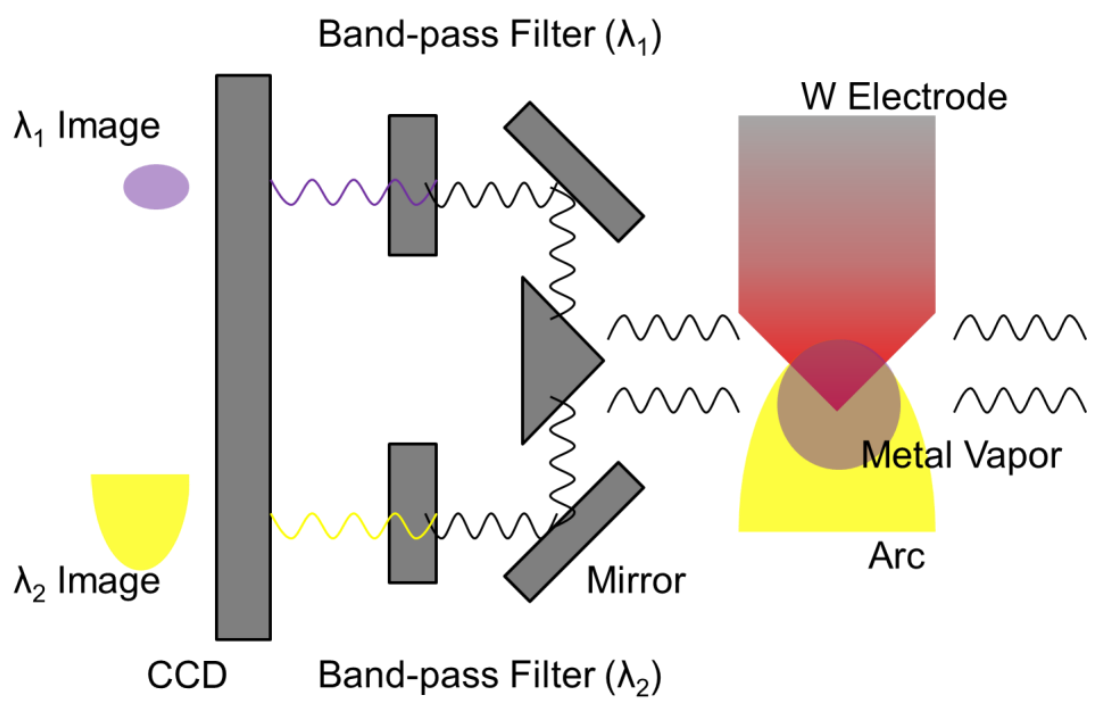

Fig. 3 Schematic diagram of the combination of the high-speed video camera and the band-pass filters.

\subsection{High-speed visualization of vapors in the arc}

Spectroscopic measurements (iHR550, Horiba Jobin Yvon) were conducted to determine the appropriate wavelengths for high-speed camera observations. High-speed video camera (FASTCAM SA-5, Photron) with the band-pass filter system was used to investigate the dynamic behavior of the vapors in the arc, as shown in Fig. 3. Emissions from argon and oxygen vapors were observed at the wavelength of $738 \mathrm{~nm}, 777 \mathrm{~nm}$, respectively. The wavelength for the tungsten vapor will be indicated in the next section. The voltage of each electrode was recorded at $1 \mathrm{MHz}$ by an oscilloscope (Scope Corder DL 850 , Yokogawa) synchronized with the high-speed camera.

\section{Results and Discussion}

Figure 4 shows the emission spectrum of the 12-phase AC arc observed at the electrode region. Line emissions from tungsten vapor, as well as that from the argon and oxygen atoms can be observed. The obtained spectrum was used to select the wavelengths of the band-pass filters for each vapor. As a result, the band-pass filter at center wavelength of 393 $\mathrm{nm}$ was selected to observe the tungsten vapor.

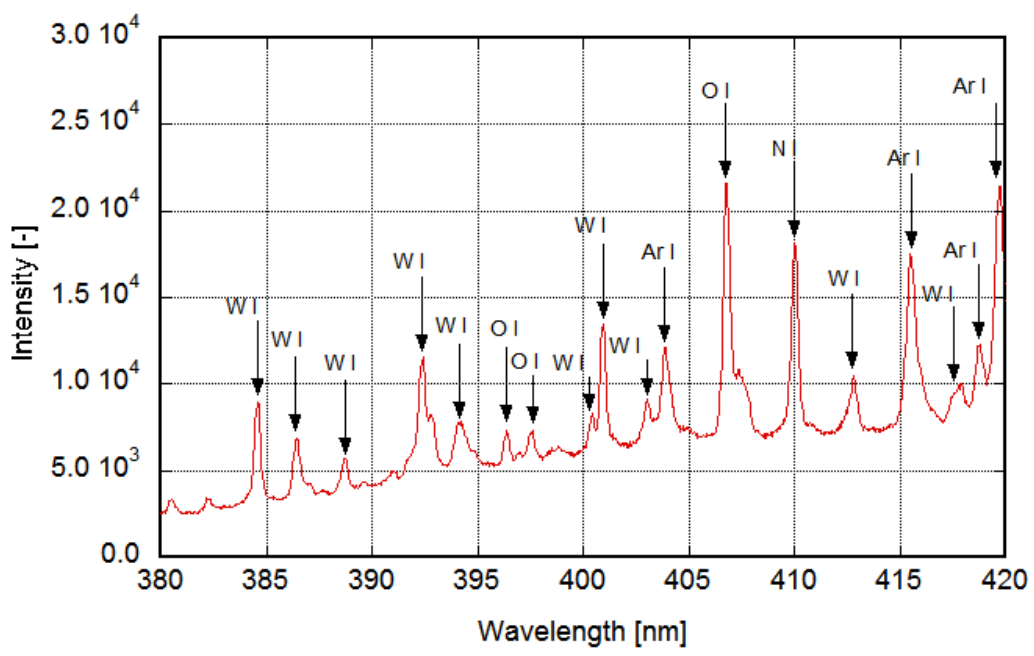

Fig. 4 Emission spectrum of the multi-phase arc in the electrode region. 
Figure 5 (a) shows the arc current waveform synchronized with the high-speed video observation in the 12-phase arc. While the representative high-speed images of the 12-phase arc for tungsten and argon vapors during one AC cycle are shown in Fig. 5 (b). The contour maps in Fig. 5 (b) indicate the distributions of the relative intensities of tungsten emission to argon emission. The relative intensities were calculated to estimate the number density of the tungsten vapor qualitatively. The indicated time in Fig. 5 (b), such as $1 \mathrm{~ms}$, corresponds to the time in Fig. 5 (a). The electrode was at the anodic period in the first half period, while at the cathodic period in the other half. The snapshots represents that the tungsten electrode started to evaporate just after the peak top of the arc current at $5 \mathrm{~ms}$ in the anodic period. Moreover, the tungsten metal vapor becomes the main species in the arc during the anodic period when it started to evaporate. However, only a small amount of tungsten evaporation was observed during the other cycle of the cathodic period. As a result, argon becomes the main species in the arc during the cathodic period.

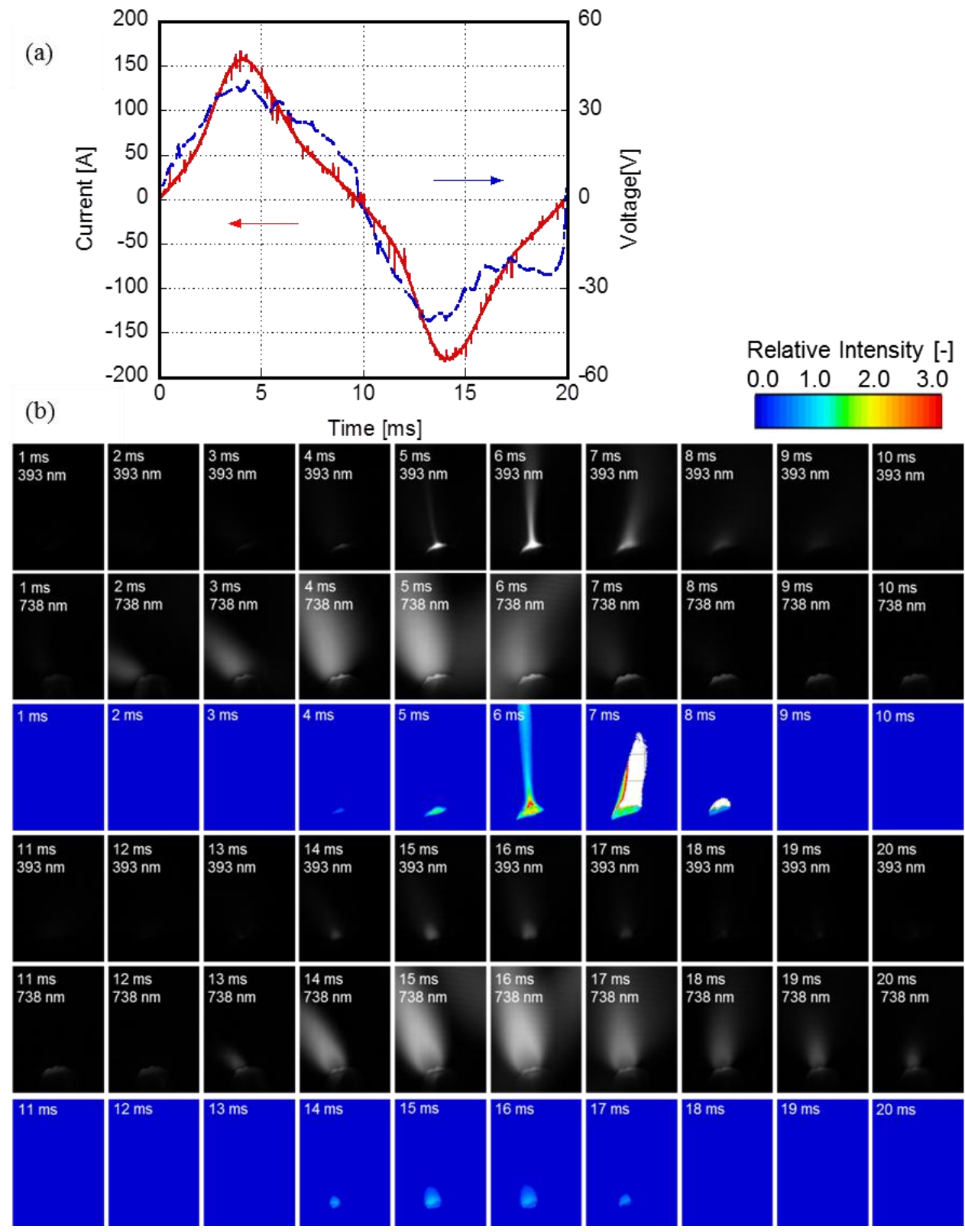

Fig. 5 Representative waveforms of the arc current and voltage in the 12-phase arc (a) and the synchronized high-speed snapshots with relative intensity distributions of tungsten to argon emissions (b). 
The difference of the electrode evaporation between the cathodic period and anodic period can be explained by the larger heat transfer to the electrode from the arc at the anodic period compared to the cathodic period. When the electron is condensed into the electrode in the anodic period, the exothermic energy of the electron is released and converted to the heat. Such released energy corresponds to the work function of the electrode. On the other hand, the electron is emitted from the electrode in the cathodic period, which process is endothermic. Consequently, the heat transfer to electrode in the anodic period is larger than that in the cathodic period. Therefore, the tungsten electrode mainly evaporates in the anodic period.

Figure 6 (a) shows the arc current waveform synchronized with the high-speed camera observation in the 2-phase arc. Figure 6 (b) shows the representative high-speed images and the distributions of the relative intensity of tungsten emission to argon emission in the 2-phase arc. In contrast to the 12-phase arc, only weak emission from tungsten vapor was observed.

(a)
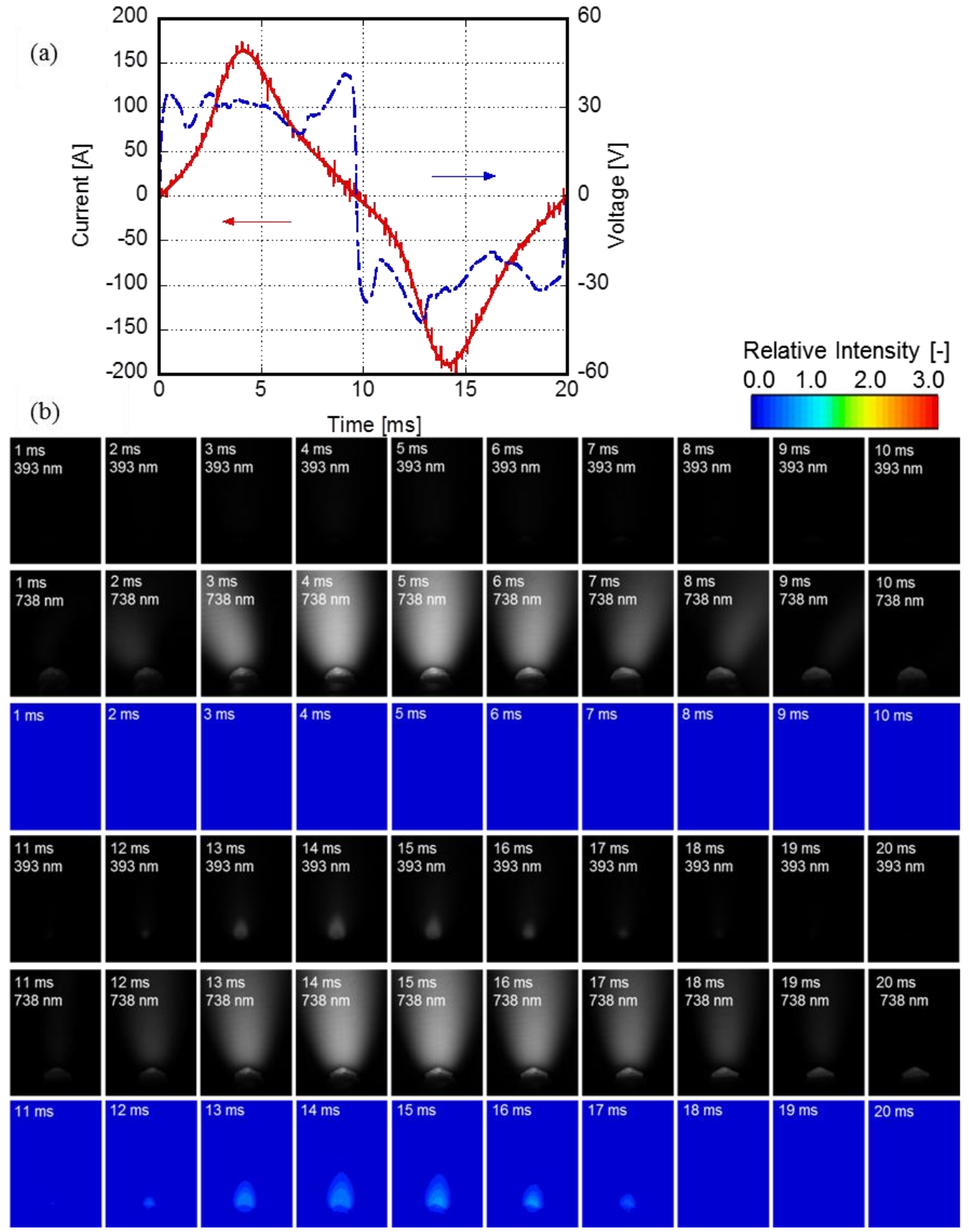

Relative Intensity [-] $\begin{array}{llll}0 & 1.0 & 2.0 & 3.0\end{array}$

Fig. 6 Representative waveforms of the arc current and voltage in the 2-phase arc (a) and the synchronized high-speed snapshots with relative intensity distributions of tungsten to argon emissions (b) 
Figure 7 shows the influence of the number of phases on the relative intensity of tungsten emission to argon emission. It should be noted that the relative intensity at $6 \mathrm{~ms}$ was selected because the tungsten evaporation was mainly observed at $6 \mathrm{~ms}$ in the anodic period. An increase of the number of the phases leads to the increase of the relative intensity. The reason for this result will be discussed in the following paragraphs.

It is already known that the melting point of the tungsten oxide is considerably lower than that of pure tungsten. Therefore, oxidation of tungsten electrode is one of the most important factors to determine the electrode erosion. Thus the influence of the number of the phases on the oxygen concentration was investigated. Figure 8 (a) shows the arc current waveform synchronized with the high-speed camera observation in the 12-phase arc. Figure 8 (b) shows the representative high-speed images and the distributions of the relative intensity in the 12-phase arc for oxygen and argon vapors during one AC cycle. The existence of the oxygen in the arc was confirmed although the argon gas was used as shield gas. The reason is considered to be the entrainment of the surrounding air. The arc is constricted by the electromagnetic force induced by the arc itself, resulting in the higher pressure in front of the electrode. Thus the strong cathode jet generates and consequently the surrounding air can be entrained into the arc region.

Figure 9 (a) shows the arc current waveform synchronized with the high-speed camera observation in the 2-phase arc. Figure 9 (b) shows the corresponding representative high-speed images and the relative intensity distributions in the 2-phase arc for oxygen emission to argon emission. The existence of the oxygen in the arc was also confirmed as well as the 12-phase arc. However the oxygen concentration in the 2-phase arc was significantly lower than that in the 12-phase arc. Figure 10 represents the influence of the number of the phases on the relative intensity of oxygen emission to argon emission. The relative intensity of oxygen emission to argon emission was increased with increasing the number of phases. The same trend was confirmed with the relative intensity of tungsten emission to argon emission.

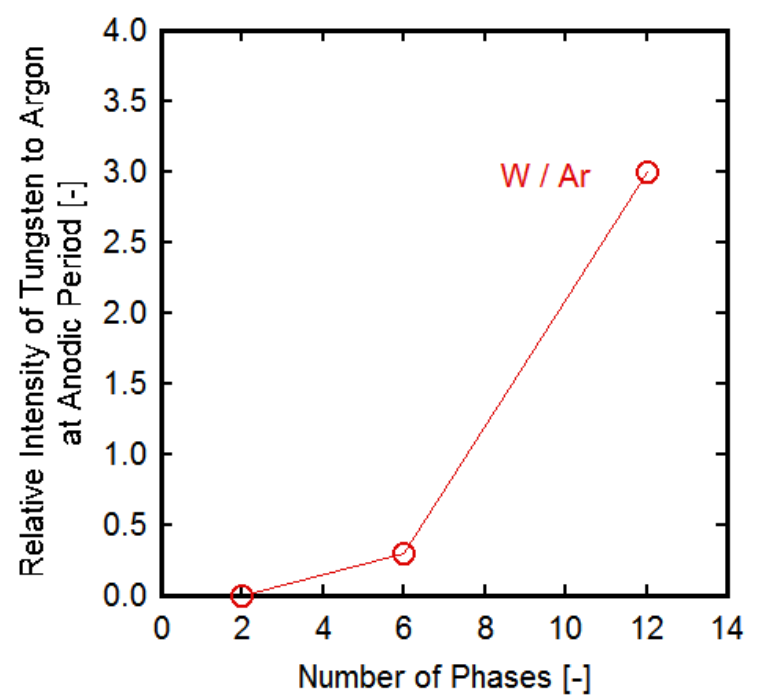

Fig. 7 Effect of the number of the phases on the relative intensity of tungsten to argon emission at the anodic period. 


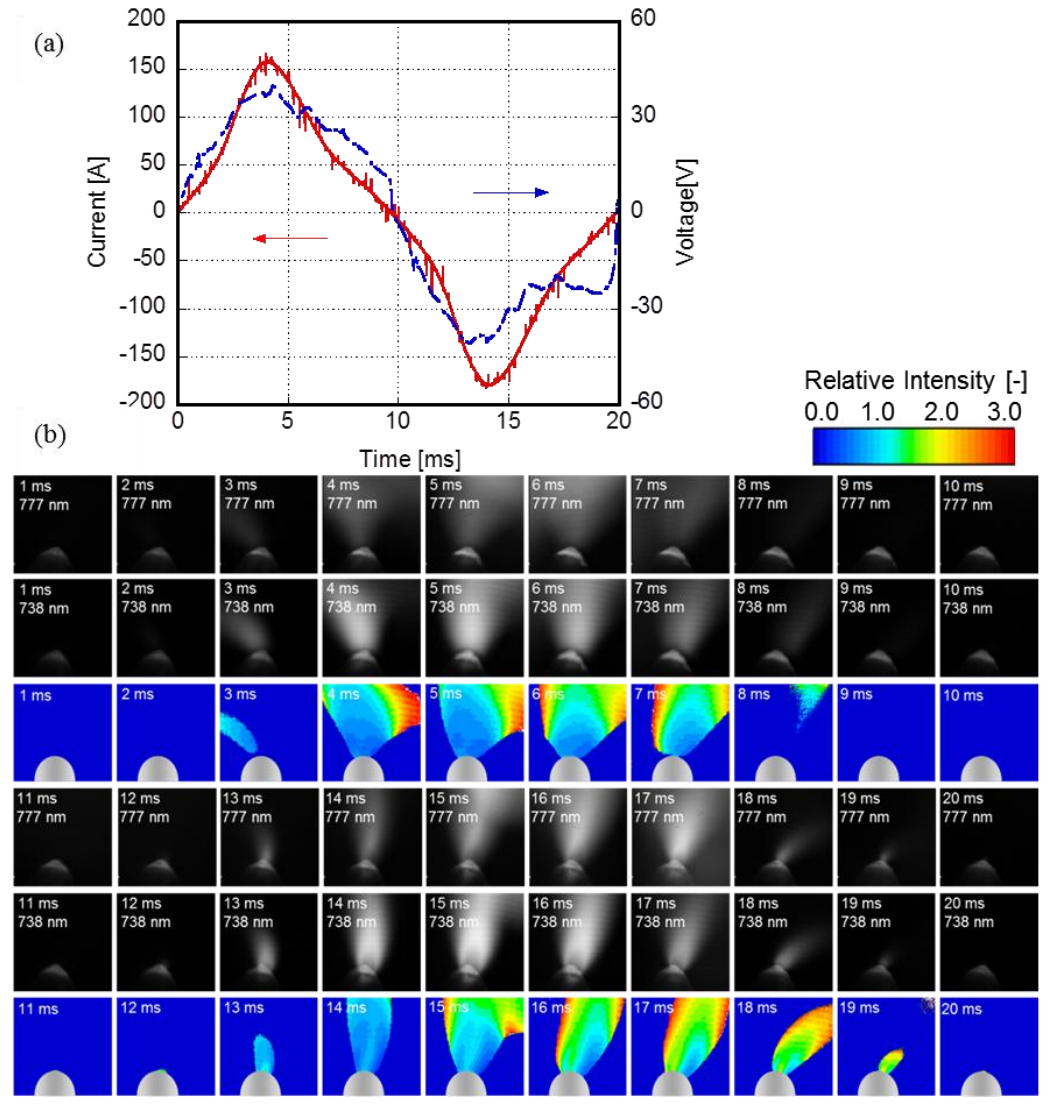

Fig. 8 Representative waveforms of the arc current and voltage in the 12-phase arc (a) and the synchronized high-speed snapshots with relative intensity distributions of oxygen to argon emissions (b).

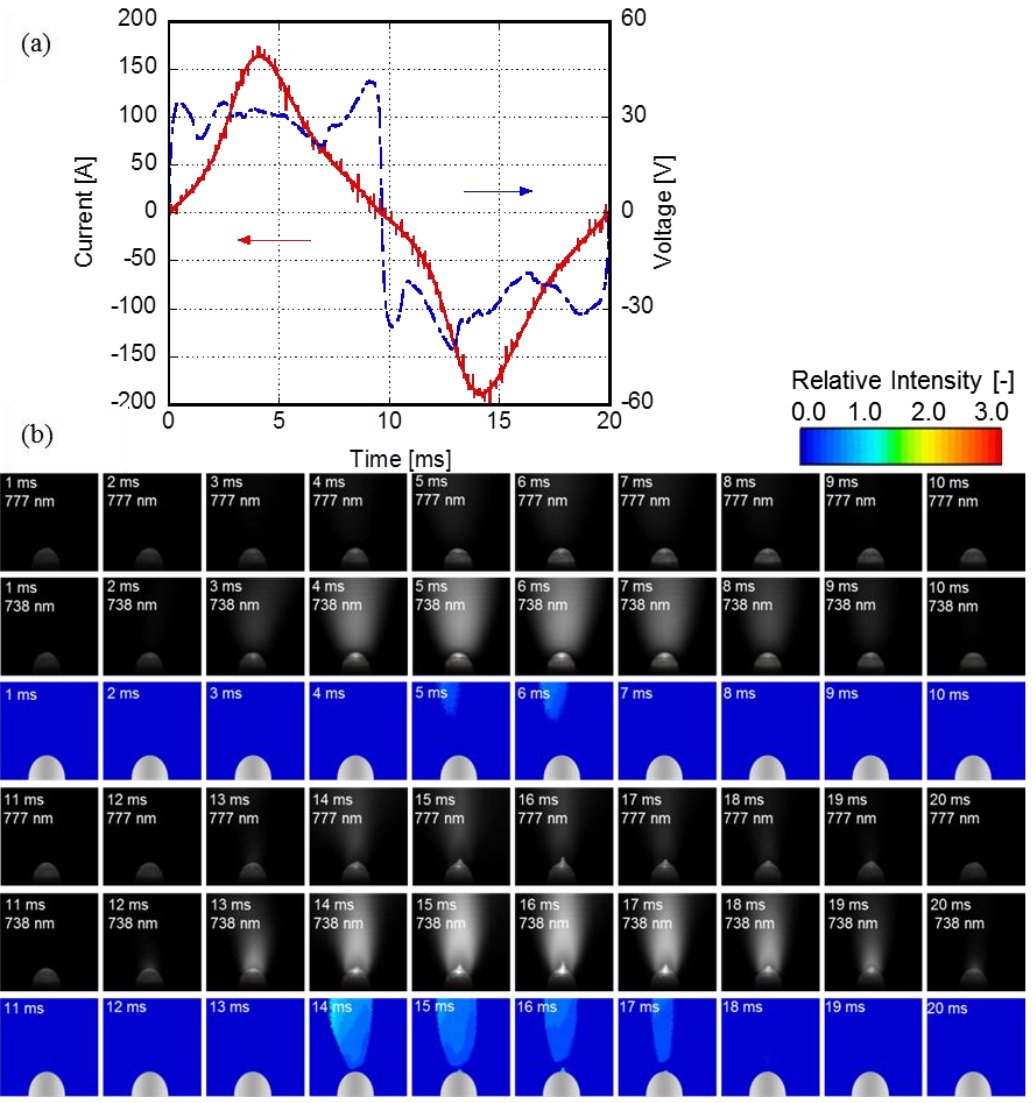

Fig. 9 Representative waveforms of the arc current and voltage in the 2-phase arc (a) and the synchronized high-speed snapshots with relative intensity distributions of oxygen to argon emissions (b). 


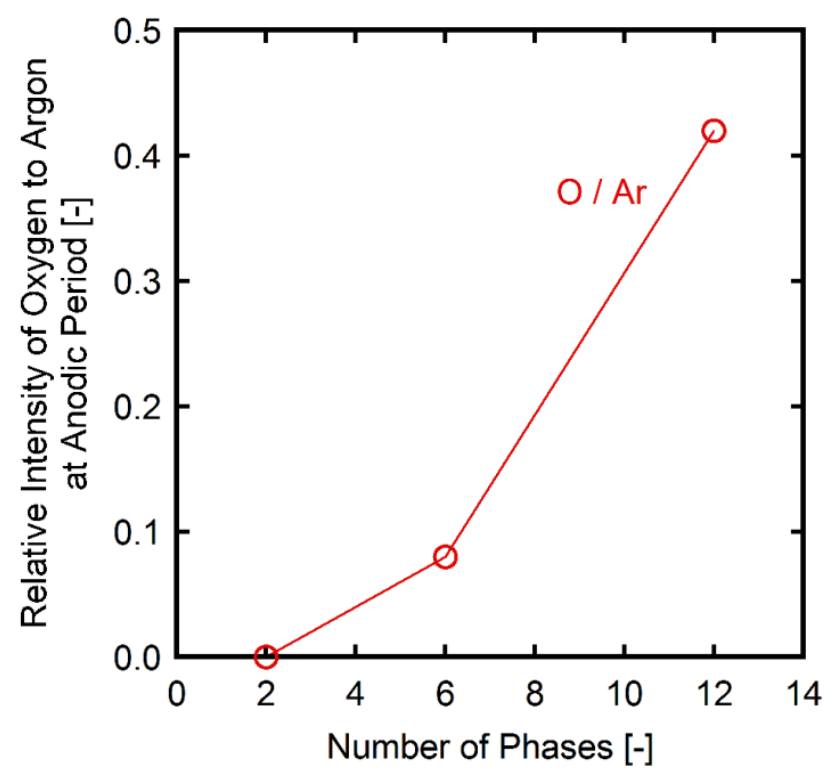

Fig. 10 Effect of the number of the phases on the relative intensity of oxygen to argon emission at the anodic period.

As mentioned in the previous report, the periodical swinging motion of the arc spot was observed in the case of the multi-phase arc ${ }^{(7,9)}$. Therefore, the arc spot motion was investigated in order to discuss the effect of the number of the phases on the oxygen concentration in the arc. Then, the high-speed images during a cycle $(20 \mathrm{~ms})$ were accumulated after binarization of each image by appropriate threshold value. These accumulated images correspond to the contour maps of the existence time of the arc, as shown in Fig. 11. From the contour maps of the arc existence time, an arc swinging angle was evaluated. Figure 12 shows the number of the phases as a relationship to the arc swinging angle. The larger number of phases leads to the increase of the swinging angle. This can be explained by the larger rotational electromagnetic field in the larger number of phases, hence the arc swings more widely. Figure 13 illustrates the model images of the oxygen entrainment into the 2-phase arc and the 12-phase arc. The ambient oxygen gas can be entrained more easily with increasing the number of phases, which resulted in the severer evaporation of the tungsten as indicated in the Fig. 7.

Further experimental investigation will be required to better understand the electrode erosion phenomena in the multi-phase AC arc system. The high-speed camera system with the appropriate band-pass filters is a strong tool to understand the dynamic behavior of the electrode phenomena.

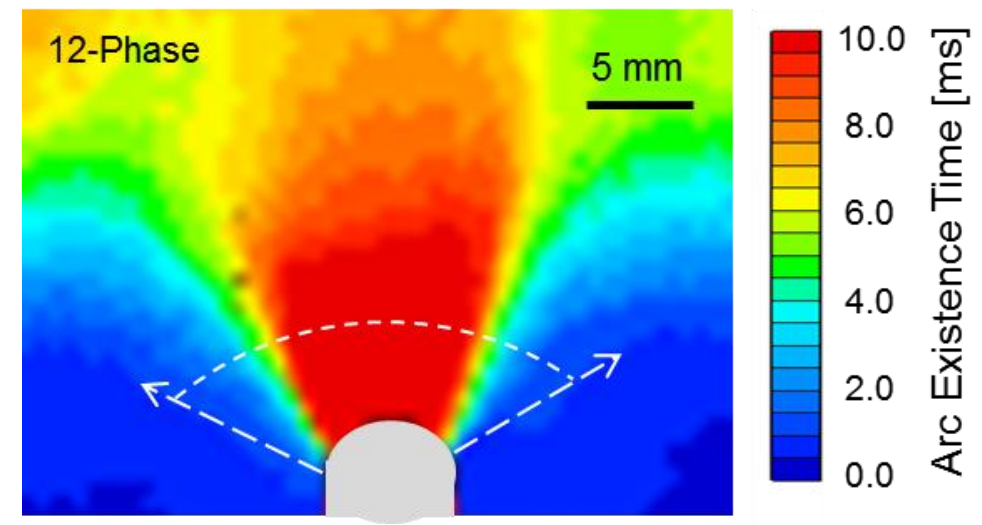

Fig. 11 Contour map of the existence time of the 12-phase arc during an AC cycle. 


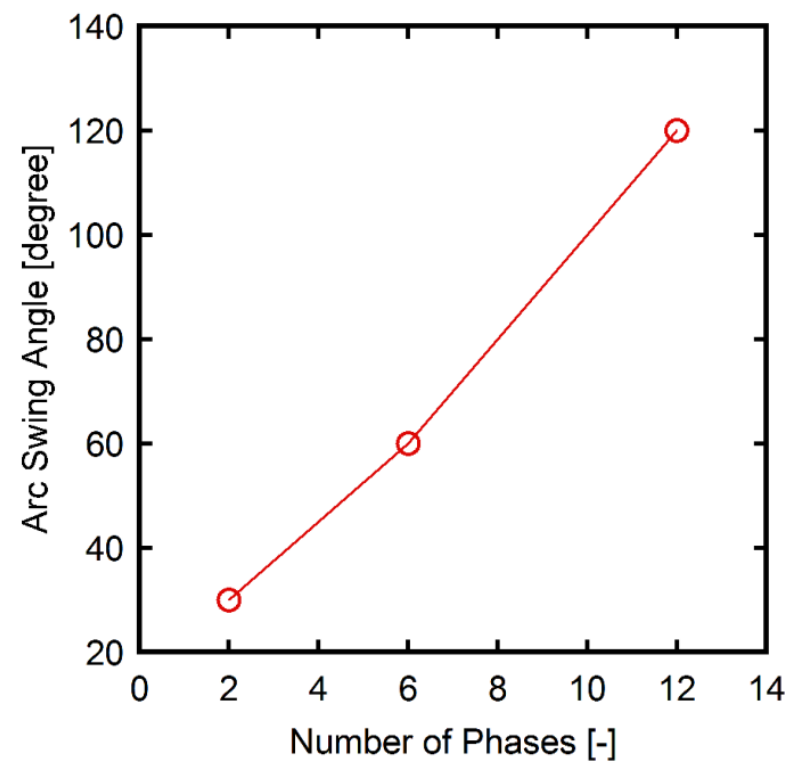

Fig. 12 Effect of the number of the phases on the arc swing angle.
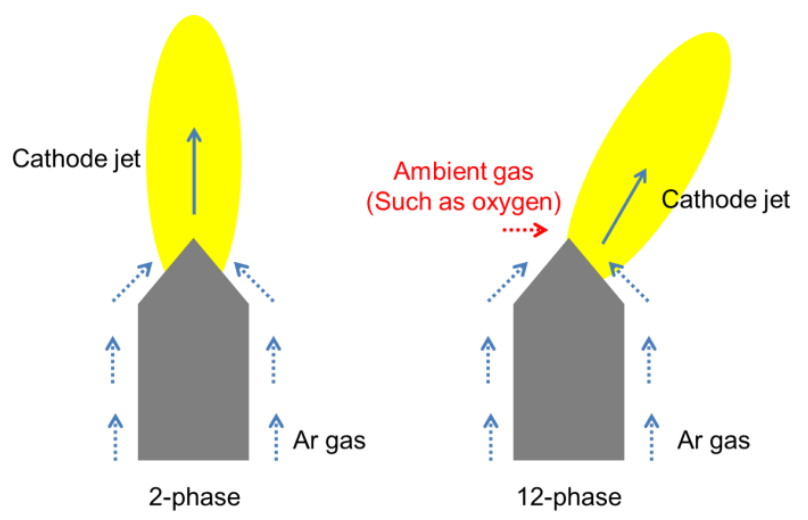

Fig. 13 The model images of the oxygen gas entrainment into the multi-phase arc.

\section{Conclusion}

The dynamic behavior of the vapors in the arc was conducted to understand the electrode erosion phenomena in the multi-phase AC arc. The high-speed camera observations with the appropriate band-pass filters were applied to achieve the purpose of the present work. The obtained results are as follows.

(1) The tungsten evaporation at the anodic period in the multi-phase AC arc was visually observed by using the high-speed camera with the band-pass filters. It was found that the tungsten started to evaporate just after the peak of the arc current in the anodic period. Then tungsten vapor becomes the main species in the arc.

(2) The oxygen concentration in the arc was increased with increasing the number of the phases due to the periodical arc-spot motion. Thus the electrode erosion becomes more severe with larger number of phases.

\section{Acknowledgement}

The financial support provided by the Strategic Development of Energy Conservation Technology Project of NEDO (New Energy and Industry Technology Development 
Organization, Japan) is gratefully acknowledged.

\section{References}

(1) Yao, Y., Watanabe, T., Yano, T., Iseda, T., Sakamoto, O., Iwamoto, M., and Inoue, S., An Innovative Energy-Saving In-Flight Melting Technology and Its Application to Glass Production, Science and Technology of Advanced Materials, Vol. 9 (2008), 025013.

(2) Yao, Y., Yatsuda, K., Watanabe, T., Funabiki, F., and Yano, T., Investigation on In-Flight Melting Behavior of Granulated Alkali-Free Glass Raw Material under Different Conditions with 12-phase AC Arc, Chemical Engineering Journal, Vol. 144 (2008), pp. 317-323.

(3) Watanabe, T., Yatsuda, K., Yao, Y., Yano, T., and Matsuura, T., Innovative In-Flight Glass-Melting Technology using Thermal Plasmas, Pure and Applied Chemistry, Vol. 82 (2010), pp. 1337-1351.

(4) Tanaka, M., Liu, Y., Tsuruoka, Y., Watanabe, T., Experimental Investigation of In-Flight Melting by Hybrid Heating of Multi-Phase Alternating Current Arc with Oxygen Burner for Alkali-Free Glass Raw Materials, Thin Solid Films, Vol. 523 (2011), pp. 67-71.

(5) Liu, Y., Tsuruoka, Y., Tanaka, M., Ichihashi, T., Yano, T., and Watanabe, T., In-Flight Melting Behavior of Different Glass Raw Materials by Hybrid Heating of Twelve-Phase AC Arc with Oxygen Burner, Thin Solid Films Vol. 519 (2011), pp. 7005-7008.

(6) Tanaka, M., Tsuruoka, Y., Liu, Y., and Watanabe, T., Investigation on In-Flight Melting Behaviour of Granulated Glass Raw Material by Multi-Phase AC Arc Plasma and Hybrid Plasma, IOP Conference Series: Material Science and Engineering Vol. 18 (2011), 112010.

(7) Tanaka, M., Tsuruoka, Y., Liu, Y., Matsuura, T., and Watanabe, T., Investigation of Multiphase AC Arc Behaviour by High-Speed Video Observation, IEEE Transactions on Plasma Science, Vol. 39 (2011), pp. 2904-2905.

(8) Tanaka, M., Tsuruoka, Y., Liu, Y., Matsuura, T., and Watanabe, T., Stability Analysis of Multi-phase AC arc Discharge for In-Flight Glass Melting, Current Applied Physics Vol. 11 (2011), pp. S35-39.

(9) Tanaka, M., Ikeba, T., Liu, Y., Choi, S., and Watanabe, T., Electrode Temperature Measurements of Multi-Phase AC Arc by High-Speed Video Camera, Journal of Physics: Conference Series, Vol. 406 (2012), 012008.

(10) Nemchinsky, V., Cathode Erosion Rate in High-Pressure Arcs: Influence of Swirling Gas Flow, IEEE Transactions on Plasma Sciences, Vol. 30 (2002), pp. 2113-2116.

(11) Nemchinsky, V. A., and Showalter M. S., Cathode Erosion Rate in High-Current High-Pressure Arc, Journal of Physics D: Applied Physics, Vol. 36 (2003), pp. 704-712.

(12) Nemchinsky V. A., Cyclic Erosion of a Cathode High-Pressure Arcs, Journal of Physics D: Applied Physics, Vol. 36 (2003), pp. 1573-1576.

(13) Peters, J., Yin, F., Borges, C. F. M., Heberlein, J., and Hackett, C., Erosion Mechanism of Hafnium Cathodes at High Current, Journal of Physics D: Applied Physics, Vol. 38 (2005), pp. 1781-1794.

(14) Colombo, V., Concetti, A., Ghedini, E., Dallavalle, S., and Vancini, M., High-Speed Imaging in Plasma Arc Cutting, Plasma Sources Science and Technology, Vol. 18 (2009), 023001 .

(15) Colombo, V., Concetti, A., Ghedini, Rotundo, F., and Dallavalle, S., Experimental Analysis of the Behaviour of High Current Electrodes in Plasma Arc Cutting During First Cyclees, Plasma Sources Science and Technology, Vol. 19 (2010), 065023.

(16) Zhou, X., and Heberlein, J., Analysis of the Arc-Cathode Interaction of Free-Burning Arcs. Plasma Sources Science and Technology, Vol. 3 (1994), pp. 564-574.

(17) Zhou, X., and Heberlein, J., Characterization of the Arc Cathode Attachment by Emission Spectroscopy and Comparison to Theoretical Predictions, Plasma Chemistry and Plasma 
Processing, Vol. 16 (1996), pp. 226-244.

(18) Zhou, X., and Heberlein, J., Experimental Investigation of Factors Affecting Arc-Cathode Erosion, Journal of Physics D: Applied Physics, Vol. 31 (1998), pp. 2577-2590.

(19) Ogawa, Y., High Speed Imaging Technique Part 1-High Speed Imaging of Arc Welding Phenomena, Science and Technology of Welding and Joining, Vol. 16 (2011), pp. 33-43.

(20) Tashiro, S., and Tanaka, M., Effect of Admixture of Metal Vapor on Cathode Surface Temperature of Plasma Torch, Surface and Coatings Technology, Vol. 202 (2008), pp. 5255-5258.

(21) Yamamoto, K., Tanaka, M., Tashiro, S., Nakata, K., Yamazaki, K., Yamamoto, E., Suzuki, K., and Murphy, A. B., Numerical Simulation of Metal Vapor Behavior in Arc Plasma, Surface and Coatings Technology, Vol. 202 (2008), pp. 5302-5305.

(22) Fujimoto, H., Tokunaga, H., and Iritani, H., A High-Powered AC Plasma Torch for the Arc Heating of Molten Steel in the Tundish, Plasma Chemistry and Plasma Processing, Vol. 4 No. 3 (1994), pp. 361-382. 